\title{
Hazardous waste management: educating industrial communities in Egypt
}

\author{
A. R. Ramadan ${ }^{1}$ \& A. H. Nadim ${ }^{2}$ \\ ${ }^{1}$ Chemistry Department, American University in Cairo, Egypt \\ ${ }^{2}$ Hazardous Waste Management Unit, Environics, Cairo, Egypt
}

\begin{abstract}
Hazardous waste management is in its initial stages in Egypt. In this regard, the need to develop awareness and capacities has been identified as a priority, particularly for the industrial establishments, constituting a major contributor to HW generation nationally. Delivery of capacity building programs for HW management to these communities has led to the development of a methodology suited to the particularities of this area in Egypt, ensuring both the effectiveness and success of these programs. Based on accumulated experience in this regard, an educational $\mathrm{CD}$ has also been developed. The elements of this methodology are presented, followed by an overall description of the CD.

Keywords: hazardous waste management, capacity building, Egypt.
\end{abstract}

\section{Introduction}

Environmental protection became a priority in Egypt over the last decade. One of the primary aims of the Egyptian Law 4 for the Environment, issued in 1994, is the reduction of pollution from industrial sources. The industrial sector in Egypt contributes to about $20 \%$ of the Egyptian GDP and employs approximately $15 \%$ of the workforce [1]. Moreover, it is the primary generator of hazardous waste (HW) [2]. Promoting and ensuring compliance to the legal stipulations in this regard is a necessity. As the implementation of proper HW management practices is relatively new in Egypt, there has been a need to build the capacity of all concerned parties with a strong focus on HW generators. In this regard, the development and delivery of targeted awareness and capacity building programs for the industrial communities in Egypt came to be recognized as an effective tool in educating such communities about their responsibilities as well as best 
practices to meet such responsibilities. This has led to the recognition of capacity building programs as a priority on the national level [3]. This paper presents a methodology developed to this end, taking into consideration the particularities and challenges of HW management in Egypt.

\section{Hazardous waste management in Egypt}

The implementation of proper HW management practices is relatively new in Egypt. The issuance of the Egyptian Law 4 in 1994, and its Executive Regulations (ER) in 1995, marked the recognition, for the first time, of HW as a source of environmental degradation [4]. However, initial enforcement activities of the stipulations of Law 4 and its ER did not place a high priority on environmental performance with regards to HW management. This was due to a number of reasons. Most significantly were the more pressing and obvious issues of air and water pollution, as well as the novelty of HW identification as a source of pollution and the practices necessary for its proper management [5].

This has led to particularities and challenges for developing and delivering capacity building programs to concerned parties. Such particularities and challenges primarily encompassed a strong sense of ambiguity and danger regarding $\mathrm{HW}$, emphasized by the less specific, yet more stringent legal stipulations particularly with regards to penalties. Furthermore, proper HW management was considered as not only necessitating thorough and detailed technical knowledge not widely available in Egypt, but also management practices forming an overall integrated system extending beyond the boundaries of the generating entity, also considered a novelty in Egypt. In this respect, an underlying overall objective of all capacity building programs for HW management in Egypt has been the elucidation of the "mystery" of HW, and the demonstration that HW identification and proper management is not only feasible, but relatively straightforward, once the necessary basic information and skills for it have been acquired.

\section{Design of programs: factors taken into account}

In general, capacity building programs are designed to increase awareness, inform, change behaviour and/or improve performance of the target audience. In this respect, the type of audience, its knowledge base, and the improvements expected as a result of the programs, are all significant factors to be taken into consideration for a successful program design and implementation. They have an impact on program content, level of details, presentation tools and the assessment and evaluation methods used.

\subsection{Type of target audience}

The target audience for HW capacity building programs varies significantly: it includes middle and top management, technical staff as well as workers of industrial establishments and HW management facilities. The variation in 
educational background, technical knowledge and skills, and the roles played within establishments are the principle factors contributing to this diversity. This variation is to be taken into account when designing and delivering programs, particularly with regards to the delivery tools: whereas lectures and presentations would form the main constituents of programs for participants from management and technical departments, little text, enhanced by visual tools such as posters and drawings as well as practical drills, are to be used for workers who, in many cases, might include people with limited literacy.

\subsection{Needs identification}

The knowledge base of the target audience and improvements expected as a result of the program (i.e. the program objectives) form the basis of needs identification for a program.

Needs $=$ Expected outcomes - Knowledge base

This in turn determines the contents of the program. For a successful program such contents necessitate that knowledge and skill gaps between what the participants know, and what is expected of them to know, be addressed so that improvements could be achieved. This affects the level of complexity of the topics and skills addressed by the program, as well as the degree of details covered.

\section{Methodology}

A number of factors were found necessary for an effective HW capacity building program in Egypt taking into account the particularities and challenges in this regard. These factors encompass the need for closely tailoring the contents to the target audience, flexibility with regard to the level of details covered during delivery, the need for audience maximum engagement, the need for enhancing the audience's realization of its "learning" progress, and the need to ensure that the increased capacities of the audience is sustained. In this respect, five components represent key features for a successful program.

\subsection{Contents}

Capacity building programs on HW management in industries must incorporate a number of fundamental topics in order to ensure their effectiveness. These include what HW is, what renders it hazardous, as well as the best practices necessary to handle such waste, i.e. the components and alternatives of a HW management system within an industrial establishment and their relation to HW management practices and services outside the establishment. However, the contents of the program must take into account two issues: the type of participants, with the identified needs for capacity building in light of their roles and responsibilities, as well as any actual specificities of the participants, such as their association to a particular industrial sector, a given geographical location or a certain size of industrial establishments. 
The type of participants would reflect on the degree of detail covered for each of the fundamental topics, but more significantly, on the focus of the program. A program targeting workers would typically focus on best practices and safety issues for handling HW containers, whereas one targeting top management would focus on the necessary steps for the implementation of proper HW management, or how to identify HW minimization options. Both programs would address the fundamental topics, but each within its own focus, resulting in somewhat different contents.

Geographical, sectorial as well as size-of-establishment specificities also impact program content. This is typically manifested not only in examples used, but also in details of HW management components and alternatives within establishments, and their relation to practices and services outside the establishments. For example, program content for small and medium textiledying establishments located within an old urban agglomeration would be different from one targeting a wide variety of industries located in a more recently developed industrial estate. Both programs would cover the fundamental topics, but the first would make use of specific examples and present collection, transportation, treatment and disposal alternatives different from what the second program would portray.

\subsection{Structure of presentations}

Flexibility with regards to level of detail covered during delivery has been found to have a number of significant advantages. Not only does it allow optimum use of allocated time, but also ensure the engagement of the participants throughout the presentation time. This flexibility is achieved by having the information covered during the presentations (power point format) structured in layers accessible through hyperlinks. In this regard, the top layer, composed of the power point slides forming the main body of the presentation, would contain the fundamental information to be covered. Links imbedded in these slides would lead to the other slides, offering clarifying details, diagrams, pictures, examples, etc., constituting the second layer of information. Within the slides of this second layer, other hyperlinks would lead to a third layer with further information deemed not crucial for clarifying the topics at hand, but useful for the knowledge base of these topics. The third layer could then possibly lead to a fourth with reference material. In this respect, the more the layers, the more added details.

For example, a presentation about storage of HW would include in its main slides the conditions for storage containers, one of which would be the presence of the necessary labels. This would lead to a second level where these labels are presented graphically, and in turn this would lead to a third level giving a descriptive account of each label and its specifications, possibly leading a fourth level addressing specific examples of HW for which these labels would be used. This structure offers the flexibility to the presenter to decide about the level of details/information covered while delivering the presentation, depending on the interest and engagement of the participants. This ensures that they would neither feel frustrated from the absence of information they consider necessary, nor bored with an overflow of details they consider irrelevant. It is important to note 
that all information of a presentation is given to the audience as handouts (electronic and/or a hardcopy) even if not dwelt on during the delivery. This is discussed further in section 4.5 below.

\subsection{Self assessment}

The effectiveness of a capacity building program is primarily based on the success of knowledge assimilation by the participants. Moreover, for HW programs, where topics are interdependent, there is a need to ensure that a clear understanding of the basics has been achieved prior to addressing more complex issues. For example a clear understating of what $\mathrm{HW}$ is must be achieved for an understanding of best practices for handling. In this respect, it was found that feedback throughout the program is necessary from capacity building recipients. This achieved through exercises carried out at a number of identified milestones at which it is deemed essential to verify participants' assimilation of information. Such exercises are usually short, typically entailing multiple choice, right and wrong questions, etc., and are carried out individually or as group work depending on the topic and the number of participants. During the exercises, several members of the program delivery team rotate between the participants, providing comments, answering queries, and taking part in any discussions. The exercises are not marked, but corrected collectively at the end the session. This was found to be more effective in allowing interactions between participants, as well as between participants and the program delivery team. The exercises are viable for technical, non-technical and less-educated participants.

These exercises were found to offer several advantages. With regards to delivery, it gives an insight to its effectiveness, allowing changes in pace, focus, use of examples and level of details to be carried out for the following sessions. More significantly, they allow the participants to self-assess their assimilation of information and gain in their knowledge base. Issues they realize still unclear can be discussed or enquired about before the end of the program, and topics they realize having been assimilated usually give them an increased enthusiasm and ensure their continued engagement in following sessions. In addition, the exercises allow a break where participants and program delivery teams can informally interact while still focusing on the topics at hand.

\subsection{Skill development}

Capacity building programs should not be limited to provide information. They must also contribute to the development of new skills for participants. A primary objective of capacity building programs is to ensure that participants are able to utilize gained knowledge in analysing situations, solving problems, and justifying their opinions. To this end, exercises, case studies, and actual site visits are used.

Exercises, different in nature from self assessment ones, primarily focus on developing skills for using existing tools aiding in $\mathrm{HW}$ identification and management. Examples include the ability of effectively using databases for identifying HW. Case studies on the other hand, simulate situations in which 
participants are likely to find themselves, requiring decisions relying on assessment and analysis of different conditions and variables. Site visits develop this further, requiring participants to note, analyse, comment on, and offer solutions for real-life HW issues in actual establishments. These different tools are carried out at different milestones within the capacity building program, and would usually be less in number than the self-assessment exercises. Typically, self assessment exercises would be carried out at the end of every session, whereas the above tools would be carried out at the end of a day (encompassing two to three sessions), or of a module (typically encompassing two days). They are typically carried out as group work, with outcomes discussed collectively at their end. These activities have been found not only useful to skill development, but also to the participants" realization of their own "learning" progress. It is noteworthy to mention that for programs addressing workers, case studies and site visits are usually replaced by practical demonstrations and drills.

\subsection{Material}

In Egypt, for HW management which is perceived as vague and confusing, material and presentation tools used for capacity building play a significant role in conveying the necessary information effectively. In this respect, these must be adapted to the type of audience, their educational background and capabilities. For top and middle management, as well as the technical staff, material and presentation tools include power point slides, printed legal and technical handouts, and other computer-aided tools such as electronic databases and flow diagrams for decision support. On the other hand, for programs targeting workers or drivers the focus is on visual material and tools. Typically for such cases, the material encompass drawings, photos and/or sketches conveying the necessary messages with minimum text. Handouts would then consist of posters and drawing of various sizes which participants can keep at hand for reference and/or hang up in the work place.

Material handed out to participants would include all information incorporated in the presentations, even if not covered in detail during the delivery of the program. In addition, reference legal and technical material would also be included. All such handouts (whether hard or electronic copies) would be compiled and clearly indexed in a manner to facilitate their later use. This is believed to sustain the developed capacities of the participants over longer spans of time. Participants would thereafter have the programs contents at hand, with the necessary basic skills to make use of them.

\section{Responses to the methodology}

The above methodology can be considered effective if it succeeds in a better development of participants' capacities, in comparison to the more widely used approach of direct lectures and presentations. In addition, its success in rendering the participants more conscious of their newly gained knowledge base and skills is also important. In this respect, both these factors have been measured for 
different capacity building programs. Typically, results of formal examinations carried out for some of the programs (as a requirement from the funding entities, or in order to obtain a certificate) would be expected to reflect a better performance for modules following this methodology. Indeed, figure 1 below demonstrates that participants to capacity building programs composed of different modules using different approaches faired better on the HW module using the above methodology. For the "Technical Training" program addressing decontamination of industrial sites, the average score of the 48 participants was about $10 \%$ higher for the HW management module than the average scores of other modules not using the above methodology. For the "Health \& Safety" program, addressing health and safety issues within industrial establishments with contamination problems, the average score of the 42 participants was about $5 \%$ higher for the HW handling module than the average scores of other modules not using the above methodology.

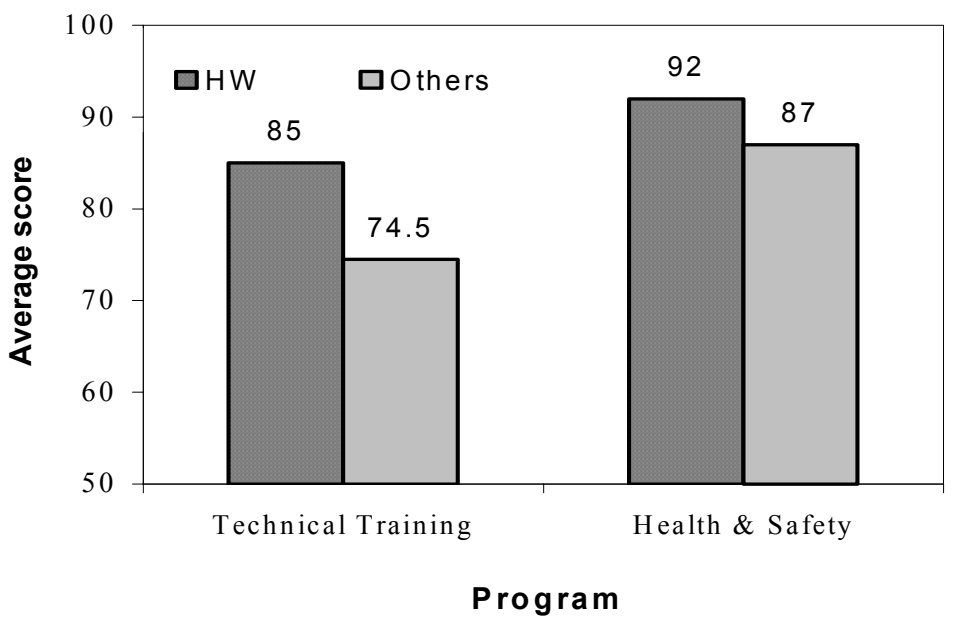

Figure 1: Examination results for the programs of "Technical Training" and "Health \& Safety" for contaminated industrial sites [6,7].

As for making participants more conscious of their newly gained knowledge base and skills, responses of program evaluation are indicative. In this respect, figure 2 summarizes responses of participants with regards to program contents, relevance, methodology, and acquired skills, for three programs addressing "HW Classification", "HW permitting", and "HW Storage". For the evaluation, the participants were requested to rate each of these parameters (among others) following a five-step scale (very satisfied, satisfied, neutral, dissatisfied, and very dissatisfied). The evaluation responses are overtly positive, with $96 \%$ to $100 \%$ of the 60 participants being satisfied or very satisfied with program content, $85 \%$ to $97 \%$ being satisfied or very satisfied with program relevance to 
their job responsibilities, $97 \%$ to $98 \%$ being satisfied or very satisfied with the methodology used for program delivery, and $84 \%$ to $96 \%$ being satisfied or very satisfied with gained skills. The lowest ratings of $84 \%$ for gained skills in the "HW Permitting" program mirrors the perception of some participants of the somewhat limited significance of the procedural skills for permitting HW activities gained during this program. Similarly, the low of $85 \%$ for relevance in the "HW Classification" program mirrors the perception of some participants of the somewhat limited relevance of classifying generated HW to their job responsibilities. Figure 3 summarizes responses of participants regarding program content, delivery methodology, and relevance to their job responsibilities, for an overall "HW Management" program, using a three-step scale (good, adequate, not suitable). Responses are overtly positive, and the rating of $100 \%$ for the delivery methodology is noteworthy.

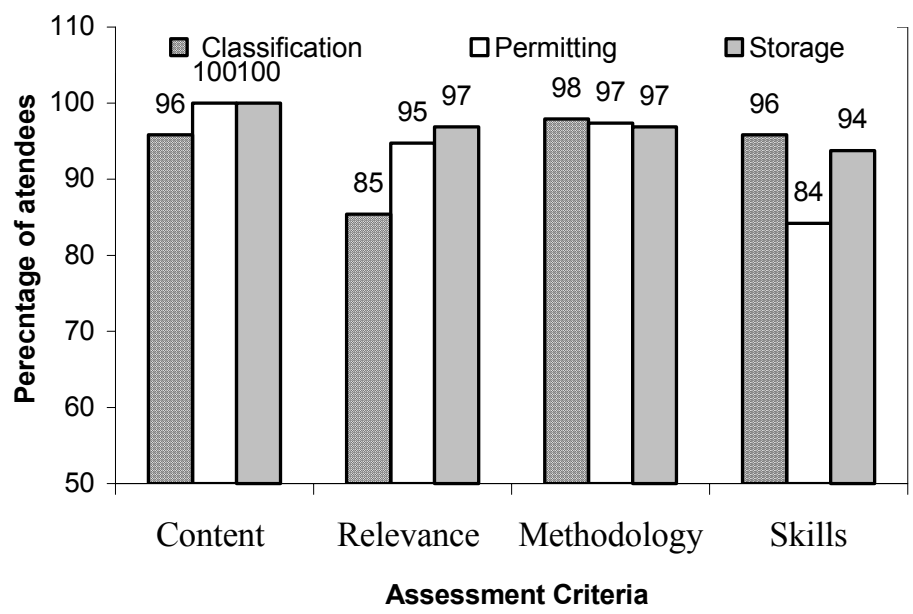

Figure 2: $\quad$ Participants feedback for three HW programs [8-10].

\section{Hazardous waste management educational CD}

With the objective of increasing awareness with regards to HW management issues in Egypt, and making use of accumulated experience in the development and delivery of capacity building programs in this regard, an educational CD was developed compiling all relevant information for HW management in Egypt. This encompasses legislation, operational guidelines, as well as references and databases. Based on experience and feedback from capacity building programs, a decision was carried out to structure the information in layers with multi access. Drop down menus, and sub-menus would allow direct access to information from an indexed list of choices. Moreover, hyperlinks imbedded within the information would allow the access to further details and interrelated topics without having to go back to the menus. This has been found to greatly facilitate navigation within the $\mathrm{CD}$. The contents encompass the topics necessary for 
promoting the operationalization of proper HW management on the national level in Egypt. In this respect, information is grouped under eight main categories: legislation, the national HW classification system, operational guidelines for HW storage, the operational guidelines for HW transportation, alternatives for HW treatment and disposal, HW minimization, HW registers, self monitoring of HW management activities in industrial establishments. This $\mathrm{CD}$, made available free of charge, was first launched in 2003, and was met with significant demand. So far, it has been widely distributed not only to industrial establishments, but also to regulating entities, and industrial non-governmental organizations and business associations.

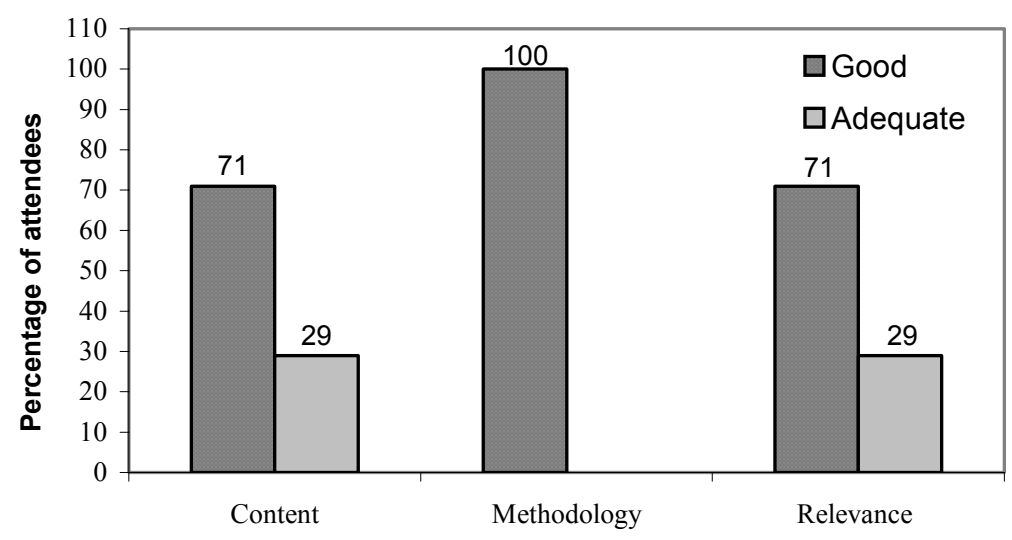

Assessment criteria

Figure 3: Participants' feedback for an overall "HW Management" program [11].

\section{Conclusion}

Capacity building is a key component to the operationalization of HW management in Egypt. Building the capacities of industrial communities, constituting a major contributor to HW generation, is faced with a number of challenges. These primarily encompass a sense of ambiguity and danger regarding $\mathrm{HW}$, with the practices for its proper management being perceived with vagueness and confusion. To develop and deliver effective programs addressing these challenges, the type of audience together with its specific needs in this regard, must be taken into account. In this respect, a methodology has been developed where program contents, structure of presentations, handout material together with exercises for self assessment and skill development ensure maximum tailoring of delivered programs to participants' needs, participants' continuous engagement, the enhancement of their realization of their "learning" progress, as well as the sustainability of their developed capacities. To further support these efforts, an educational CD on HW management was developed and widely distributed. 


\section{References}

[1] Egyptian Pollution Abatement Project, Strategy and Action Plan for Cleaner Production in Egyptian Industry, Egyptian Environmental Affairs Agency, 2004.

[2] Egyptian Pollution Abatement Project, Hazardous Waste Management Manual for Industry, Egyptian Environmental Affairs Agency, 2003.

[3] National Environmental Action Plan (NEAP), Egyptian Environmental Affairs Agency, 2002.

[4] Executive Regulations of Law 4/1994 for the Environment, Chapter 2, 1995.

[5] Ramadan, A.R, Nadim A.H, Hazardous Waste Management in Egypt: Status and Challenges, Proc. of Waste Management and the Environment II, eds. V. Popov, H. Itoh, C.A. Brebbia and S. Kungolos, WIT Press: Southampton and Boston, pp. 125-135, 2004.

[6] LIFE Lead Pollution Clean-up in Qalyoubia, Technical Remediation Training, Egyptian Environmental Affairs Agency, 2005.

[7] LIFE Lead Pollution Clean-up in Qalyoubia, Health \& Safety Training, Egyptian Environmental Affairs Agency, 2005.

[8] Development Training II (DT2), How to Characterizel Classify Hazardous Waste, Egyptian Environmental Affairs Agency, 2002.

[9] Development Training II (DT2), Permitting of Hazardous Waste, Egyptian Environmental Affairs Agency, 2002.

[10] Development Training II (DT2), On-site Storage of Hazardous Waste, Egyptian Environmental Affairs Agency, 2002.

[11] Hazardous Waste Management Project in Alexandria, Hazardous Waste Management, Egyptian Environmental Affairs Agency, Alexandria Governorate, 2003. 